\title{
Endovascular Management of Great Vessel Injury Following Lumbar Microdiscectomy
}

\author{
Hee-Kwon Park ${ }^{1}$, Woo Jin Choe ${ }^{1}$, Young-Cho Koh ${ }^{1}$, Sang Woo Park ${ }^{2}$ \\ Departments of Neurosurgery ${ }^{l}$, Radiology ${ }^{2}$, Konkuk University College of Medicine, Seoul, Korea
}

Great vessel injury is a rare but well-known complication of lumbar disc surgery, which may result in acute or fatal outcomes of delayed diagnosis. Thus, early detection and proper management is vital. The authors report a case of retroperitoneal hemorrhage with arteriovenous fistula and pseudoaneurysm after lumbar microdiscectomy. The patient was successfully managed by endovascular intervention using a stent graft. Endovascular repair is a minimally invasive and efficient treatment modality with considerably low morbidity and mortality.

Key Words: Lumbar disc surgery • Vascular injury

\section{INTRODUCTION}

Great vessel injury is a rare but fatal complication that can occur during lumbar disc surgery. Great vessel injury can result in massive retroperitoneal hemorrhage or present as a delayed pseudoaneurysm or arteriovenous fistula (AVF) $)^{2,3,6,8,9,11)}$. Despite a very low incidence $(0.016-0.17 \%)$, the reported mortality rate ranges from $15 \%$ to $100 \%$, and this complication may elicit serious medicolegal problems ${ }^{2,4,5,7,9,10,12)}$. In cases of great vessel injury, early detection and management is critical to ensure patient survival ${ }^{1,4,7}$. Major vascular surgery is often required to prevent fatality; however, procedure-related morbidity and mortality must also be considered ${ }^{4,5,10)}$.

We describe the case of a woman who sustained a common iliac artery and vein injury after L4-5 lumbar microdiscectomy. Retroperitoneal bleeding with an arterial pseudoaneurysm was successfully treated by an endovascular stent graft. No major complications were reported.

\section{CASE REPORT}

A 48-year-old woman referred to hospital for radiating pain

- Received: October 31, 2013 - Revised: November 25, 2013

- Accepted: November 28, 2013

Corresponding Author: Woo Jin Choe, MD, PhD

Department of Neurosurgery, Konkuk University Medical Center 120-1,

Neungdong-ro, Gwanjin-gu Seoul 143-729, Korea

Tel: +82-2-2030-7625, Fax: +82-2-2030-7359

E-mail: slnsman@chol.com

$\otimes$ This is an Open Access article distributed under the terms of the Creative Commons Attribution Non-Commercial License (http://creativecommons.org/ licenses/by-nc/3.0/) which permits unrestricted non-commercial use, distribution, and reproduction in any medium, provided the original work is properly cited.

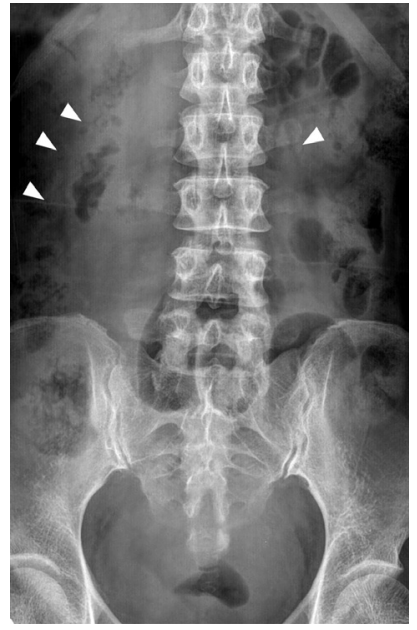

Fig. 1. Postoperative radiographs show blurring of the right psoas shadow (arrowheads). Hemoglobin levels dropped from $13.5 \mathrm{~g} / \mathrm{dL}$ to $8.5 \mathrm{~g} / \mathrm{dL}$ postoperatively without any clinical manifestations.

in her lower back and right leg. Symptoms appeared approximately two months previously. Motor weakness and sensory deficits were not observed. Magnetic resonance imaging of the lumbar spine showed a ruptured disc in L4-5. Conservative management was not effective; therefore, lumbar microdiscectomy was performed. The herniated disc was removed successfully. Trivial hemorrhage occurred near the end plate that was easily controlled with saline irrigation. Patient vital signs were stable during the operation and immediate postoperative period, and the radiating pain disappeared with no evidence of neurological deficit. Although no symptoms such as abdominal cramps were noted, the postoperative hemoglobin level decreased from $13.5 \mathrm{~g} / \mathrm{dL}$ initially to $8.6 \mathrm{mg} / \mathrm{dL}$ postoperatively. Postoperative radiographs showed a blurred margin of the psoas shadow (Fig. 1). With the suspicion of postoperative hemorrhage, emergent abdominal ultrasonography and computed tomography $(\mathrm{CT})$ were performed. Imaging 


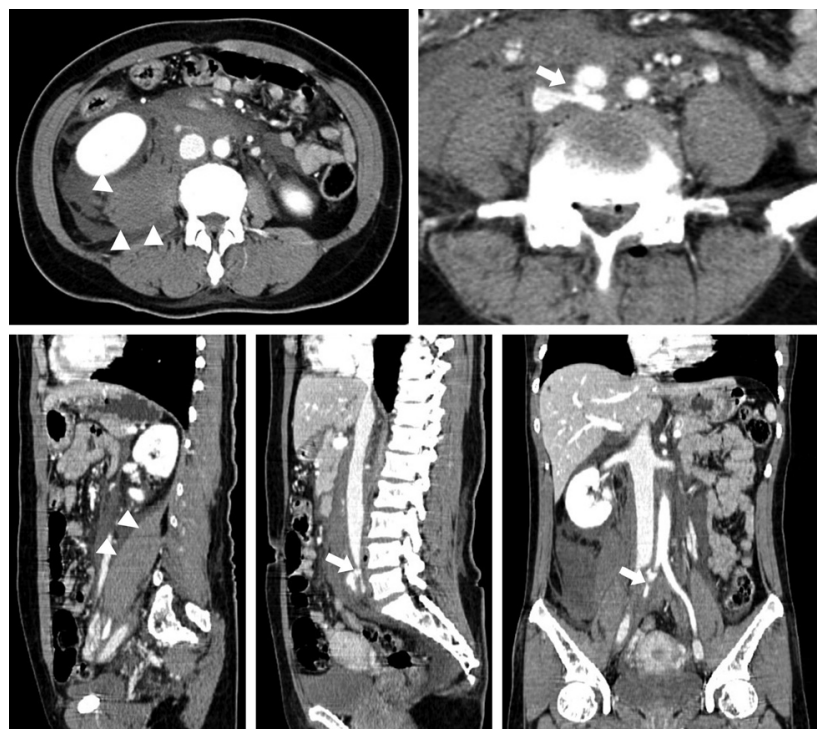

Fig. 2. Emergent enhanced abdominal computed tomography scans reveal a retroperitoneal hematoma (arrowheads). A pseudoaneurysm (arrow) between the common iliac artery and iliac vein is seen in front of the L4-5 intervertebral disc space.

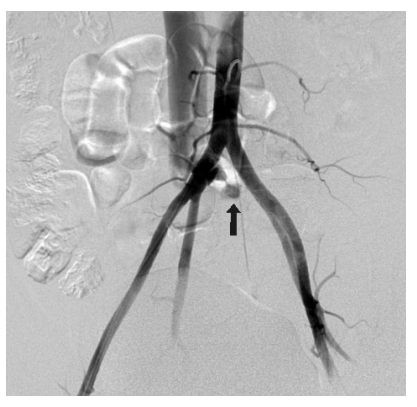

Fig. 3. Angiography demonstrates the formation of an arteriovenous fistula between the right common iliac artery and right common iliac vein with a pseudoaneurysm (black arrow).

studies revealed a large retroperitoneal hematoma and a pseudoaneurysm near the right common iliac artery and right common iliac vein (Fig. 2).

Vital signs were monitored, with blood pressure at 110/70 $\mathrm{mmHg}$ and heart rate at 70 beats $/ \mathrm{min}$. While vital signs were stable, an endovascular intervention was planned. Aortography demonstrated a traumatic AVF between the right common iliac artery and the right common iliac vein with a $1 \mathrm{~cm}$-sized pseudoaneurysm (Fig. 3). The AVF was obliterated using a SEAL stent graft (S\&G Biotch Inc., Seoul, Korea) and a $9 \times$ $4 \mathrm{~cm}$-sized balloon at the right common iliac artery (Fig. 4). After successful obliteration of the AVF, no evidence of the pseudoaneurysm was seen on angiography. The patient recovered without any complications or sequelae. The patient was discharged on postoperative day 8 . No retroperitoneal hemorrhage was identified on the 3-month follow-up CT scan (Fig. 5). The patient was given a 1-year supply of clopidogrel, and was stable and asymptomatic 3 years after the surgery.
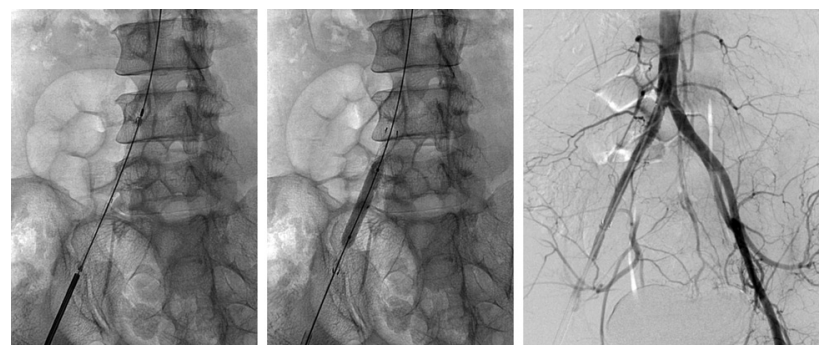

Fig. 4. The fistula was obliterated using a stent graft and a balloon at the right common iliac artery. Only arterial side laceration was obliterated, and fistula with pseudoaneurysm was not visible on angiography. Vascular bleeding was stopped via tamponade effect of the hematoma.
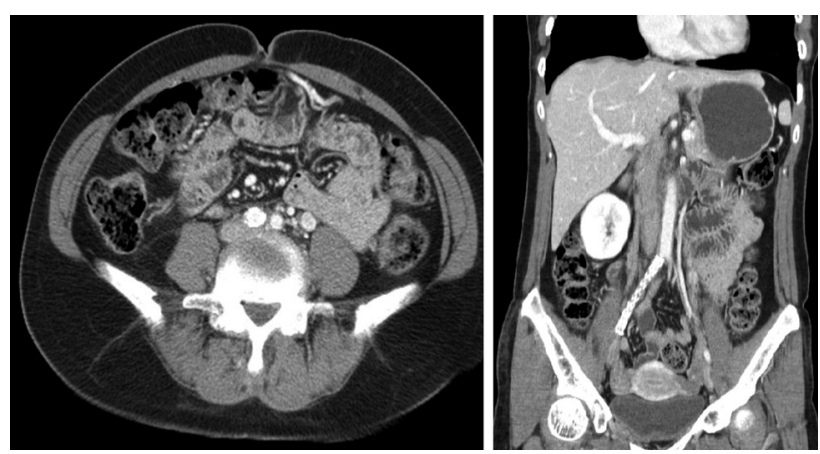

Fig. 5. Computed tomography obtained three months after stent graft insertion shows absorption of retroperitoneal hemorrhage and well maintained blood flow thorough the right iliac artery. The arteriovenous fistula with pseudoaneurysm is not observed.

\section{DISCUSSION}

Major vessel injury is a rare but well-known complication in lumbar disc surgery. Despite advances in modern microscopic surgical techniques and laser-assisted surgery, there has been no significant decrement in the incidence since $1945^{2,3,5}$, ${ }^{9,10)}$. Since many cases remain undetected or are not reported, the true incidence of major vessel injury may be higher than what is reported in the literature.

The common iliac artery and common iliac vein are located anterior to the L4-L5 lumbar and L1 sacral vertebral bodies, which is where great vessel injury most frequently occurs ${ }^{1,4)}$. Great vessel injury is the most frequent injury that occurs when operating on the L4-5 lumbar segments, ${ }^{6,9)}$. The injury is caused by the perforation of the anterior spinal ligament while attempting complete removal of disc material using a rongeur. The common iliac vessels and the disc are usually separated by the anterior spinal ligament alone at the lower lumbar spinal level. Disc degeneration may weaken this ligament and alter the relationship between the ligament and the 
adjacent vascular structures ${ }^{2,3)}$.

The clinical course of vascular injuries can vary and can be categorized into acute, subacute, and chronic. Acute blood loss may occur due to damage caused by a severe arterial laceration and lead to sudden hypovolemia ${ }^{10-12)}$. Diagnosis of AVFs or pseudoaneurysms can be delayed because of unusual clinical symptoms and a lack of specific warning signs.

Vessel injuries may be detected quickly, especially in cases of profuse arterial bleeding and hypotension with tachycardia. However, as in the present case, bleeding may be minimal or go undetected in half of the injuries, ${ }^{6,7}$. Even when massive hemorrhage occurs, blood usually collects in the retroperitoneal space, and blood pooling in the disc space may not be recognized in the surgical field. Moreover, the prone position in which the patients are operated may confer a degree of vascular compression during surgery, and as such, this may temporarily tamponade any vascular tears ${ }^{7,9)}$. Surgeons should suspect vessel injury if hemorrhage or fat tissue is observed during a lumbar discectomy even when vital signs are stable.

After a vessel injury, the duration of detection and the treatment approach are associated with the mortality rate $e^{1,25,7,12}$. When the vessel injury is recognized intraoperatively and signs of life-threatening hypovolemic shock are observed, vigorous volume replacement and urgent repair of vascular laceration should be considered. In arterial injuries with critical bleeding, the mortality rate increases to $100 \%$ when surgery is not immediately performed ${ }^{4,6,8}$. Although the injury is not confirmed during surgery and the vital signs are stable, insignificant symptoms such as abdominal distension, pain, palpitation, unexplainable anemia, and blurring of the psoas muscle on radiographs are possible predictors of vessel laceration. Abdominal ultrasonography and CT angiography are the preferred methods of diagnosis in confirming vessel injury and retroperitoneal hematoma ${ }^{11,12)}$. When diagnosed, conventional angiography and interventional procedure should be performed.

In the past, vascular injury following a lumbar discectomy was treated by direct surgical repair of the damaged vessels ${ }^{9,10)}$. Although the current outcomes are considered good, surgical repair of traumatic or postoperative vascular injury may result in considerable blood loss and complications, ${ }^{4,10)}$. Endovascular techniques are an alternative method of managing the vascular injury following lumbar disc surgery. Endovas- cular techniques using a stent graft to occlude AVF or leakage show satisfactory bleeding control with few complications ${ }^{1,4,7,10,11)}$. The advantages of these procedures are the absence of a lower abdominal incision, minimal blood loss, and reduced depth and length of anesthesia, which consequently allows for shorter hospitalization periods compared with conventional surgery. It can also reduce perioperative morbidity and mortality in hemodynamically unstable patients. However, proper facili- ties and preparation of stent grafts and other materials are mandated for endovascular intervention ${ }^{7}$.

Delayed onset of symptoms or signs may occur in patients who undergo lumbar disc surgery because of an AVF or pseudoaneurysm ${ }^{2,8,11)}$. Swelling of the legs, fatigue, shortness of breath, cardiac failure, classic machinery bruit in the abdomen, and delayed bleeding may lead to the diagnosis of $\mathrm{AVF}^{6,8)}$. Most isolated vascular injuries of the great retroperitoneal veins remain clinically silent because of a tamponade effect of a perivascular hematoma ${ }^{1)}$. Therefore, the treatment of stable AVFs or pseudoneurysms should not be delayed, and only arterial side sealing would be sufficient, as in the present case.

\section{CONCLUSION}

Vascular injury following lumbar disc surgery necessitates early recognition, diagnosis, and prompt surgical repair for prevention of fatal outcomes. An endovascular repair using a stent graft is a minimally invasive and efficient treatment modality with low morbidity.

\section{REFERENCES}

1. Bierdrager E, Van Rooij WJ, Sluzewski M: Emergency stenting to control massive bleeding of injured iliac artery following lumbar disk surgery. Neuroradiology 46:404-406, 2004

2. Erku B, Unlu Y, Kaygin MA, Colak A, Erdem AF: Iatrogenic vascular injury during to lumbar disc surgery. Acta Neurochir 149:511-515, 2007

3. Jeon $\mathrm{SH}$, Lee $\mathrm{SH}$, Choi WC: Iliac artery perforation following lumbar discectomy with microsurgical carbon dioxide laser. Spine 32:124-125, 2007

4. Jin SC, Park SW, Cho DS: Management of proximal iliac artery injury during lumbar discectomy with stent graft. J Korean Neurosurg Soc 51:227-229, 2012

5. Lee CK, Kim SC, Kim SH: Common iliac vessel injury after lumbar discectomy. Korean J Spine 8:229-131, 2011

6. Murat D, Merih I, Mevlut P, K. Hakan Y: Nightmare of lumbar disc surgery: iliac artery injury. Clinical Neurology and Neurosurgery 108:174-177, 2006

7. Nam TK, Park SW, Shim HJ, Hwang SN: Endovascular treatment for common iliac artery injury complicating lumbar disc surgery: limited usefulness of temporary balloon occlusion. J Korean Neurosurg Soc 46:261-264, 2009

8. Prabhakar H, Bithal PK, Dash M, Chaturvedi A: Rupture of aorta and inferior vena cava during lumbar disc surgery. Acta Neurochir (Wien) 147:327-329, 2005

9. Bingol H, Cingoz F, Yilmaz AT, Yasar M, Tatar H: Vascular complications related to lumbar disc surgery. J Neurosurg (Spine 3) 100:249-253, 2004

10. van Zitteren M, Fan B, Lohle PN, de Nie JC, de Waal Malefijt J, Vriens PW, et al: A shift toward endovascular repair for vas- 
cular complications in lumbar disc surgery during the last decade. Annals Vascular Surgery 27:810-819, 2013

11. Wu CY, Hung YN, Liu YH, Ko PJ: Endovascular treatment of iatrogenic iliac artery disruption in lumbar disc surgery. Annals Vascular Surgery 23:255.e7-255.e11, 2009
12. Papadoulas S, Konstantinou D, Kourea HP, Kritikos N, Haftouras N, Tsolakis JA: Vascular injury complicating lumbar disc surgery. A Systematic Review. Eur J Vasc Endovasc Surg 24:189195,2002 